\title{
Chiral extrapolation of the $\sigma$ and $\rho$ mesons from dispersion relations and Chiral Perturbation Theory
}

\author{
G. Ríos*, A. Gómez Nicola*, C. Hanhart ${ }^{\dagger}$ and J. R. Pélaez* \\ ${ }^{*}$ Dept. Física Teórica II. Universidad Complutense, 28040, Madrid. Spain \\ ${ }^{\dagger}$ Institut für Kernphysik (Theorie), Forschungzentrum Jülich, D-52425 Jülich, Germany
}

\begin{abstract}
We review our recent study of the pion mass dependence of the $\rho$ and $\sigma$ resonances, generated from one-loop $S U(2)$ Chiral Perturbation Theory (ChPT) with the Inverse Amplitude Method (IAM). In order to properly account for the Adler zero region, we also review the recently obtained modified version of the IAM; which is based on analyticity, elastic unitarity and ChPT at low energies, thus yielding the correct pion mass dependence of the resonance pole positions up to next-to-leading order in ChPT. As main results we find that the $\rho \pi \pi$ coupling constant is almost $m_{\pi}$ independent and that $M_{\rho}$ shows a smooth $m_{\pi}$ dependence while that of the $\sigma$ shows a strong non-analyticity. These findings are important for studies of the meson spectrum on the lattice.
\end{abstract}

Keywords: Chiral extrapolations, Dispersion relations, light mesons

PACS: $14.40 . \mathrm{Cs}, 12.39 . \mathrm{Fe}, 11.55 . \mathrm{Fv}, 13.75 . \mathrm{Lb}$

\section{INTRODUCTION}

Light hadron spectroscopy lies beyond the realm of perturbative QCD. Although lattice QCD provides, in principle, a rigorous way to extract non-perturbative quantities, present calculations use relatively high quark masses, at least for studies of scalar mesons, i. e., [1, 2]. Thus, appropriate extrapolation formulas are called for. Chiral Perturbation Theory (ChPT) [3] provides such extrapolations, since it is built as an expansion in momenta and masses, generically $O\left(p / 4 \pi f_{\pi}\right)^{2}$, of a Lagrangian involving the Goldstone Bosons of the QCD chiral symmetry breaking (pions), compatible with all QCD symmetries. ChPT is renormalized order by order by absorbing loop divergences in the parameters of higher order counterterms (low energy constants - LECs), which are the coefficients of the energy and mass expansion, so they have no quark mass dependence. Their values depend on the QCD dynamics, and have to be determined from experiment. In SU(2)-ChPT $\pi \pi$ scattering only four LECs appear, denoted $l_{i}$.

The ChPT expansion provides a systematic and model independent description of how the observables depend on QCD parameters, like the quark masses, and this can be implemented systematically up to the desired order in the ChPT expansion.

We review here our recent derivation of a modified version of the IAM [4]; based on dispersion theory, unitarity and ChPT to next-to-leading order (NLO), which we use to predict the quark mass dependence of the $\sigma$ and $\rho$ mesons [5].

We focus only on the $\sigma$ and the $\rho$, so it is enough to work with the lightest quarks $u, d$ in the isospin limit with a mass $\hat{m}=\left(m_{u}+m_{d}\right) / 2$. Since $m_{\pi}$ is given by $m_{\pi}^{2} \sim \hat{m}+\ldots$ [3], studying the $\hat{m}$ dependence is equivalent to study the $m_{\pi}$ dependence. 


\section{UNITARIZATION AND DISPERSION THEORY}

The $\sigma$ and $\rho$ resonances appear as poles in the second Riemann sheet of the $(I, J)=(0,0)$ and $(1,1)$ partial waves of isospin $I$ and angular momentum $J$ respectively. For these partial waves, elastic unitarity implies, for physical values of $s$ :

$$
\operatorname{Im} t(s)=\sigma(s)|t(s)|^{2} \Rightarrow \operatorname{Im}\left(t(s)^{-1}\right)=-\sigma(s), \quad \text { with } \quad \sigma(s)=2 p / \sqrt{s},
$$

where $s$ is the Mandelstamm variable and $p$ is the center of mass momentum. Consequently, the imaginary part of the inverse amplitude is known exactly. ChPT amplitudes, being an expansion $t=t_{2}+t_{4}+\cdots$ with $t_{k}=O\left(p^{k}\right)$, satisfy Eq. (1) just perturbatively:

$$
\operatorname{Im} t_{4}(s)=\sigma(s)\left|t_{2}(s)\right|^{2}, \quad \Rightarrow \quad \operatorname{Im} t_{4}(s) / t_{2}(s)^{2}=\sigma(s),
$$

and cannot generate poles. Therefore the resonance region lies beyond the reach of standard ChPT. However, it can be reached by combining ChPT with dispersion theory either for the amplitude [6] or the inverse amplitude through the IAM [7, 8, 9].

The elastic IAM [4] uses the ChPT series and elastic unitarity to evaluate a dispersion relation for the inverse amplitude. The analytic structure of $1 / t$ consists on a right cut from threshold to $\infty$, a left cut from $-\infty$ to $s=0$, and possible poles coming from zeros of $t$. For scalar waves, $t$ vanishes at the so called Adler zero, $s_{A}$, that lies on the real axis below threshold, thus within the ChPT region of applicability. Its position can be obtained from the ChPT series, i.e., $s_{A}=s_{2}+s_{4}+\cdots$, where $t_{2}$ vanishes at $s_{2}, t_{2}+t_{4}$ at $s_{2}+s_{4}$, etc.

We write then a once subtracted dispersion relation for the inverse amplitude, where we have chosen the subtraction point to be the Adler zero:

$$
\frac{1}{t(s)}=\frac{s-s_{A}}{\pi} \int_{R C} d s^{\prime} \frac{\operatorname{Im} 1 / t\left(s^{\prime}\right)}{\left(s^{\prime}-s_{A}\right)\left(s^{\prime}-s\right)}+L C(1 / t)+P C(1 / t),
$$

where "LC" stands for a similar integral over the left cut and "PC" stands for the contribution of the pole at the Adler zero. Since $t_{2}$ is real on the real axis and $t_{4}$ has the same analytic structure as $t$, we can similarly write

$$
\frac{t_{4}(s)}{t_{2}(s)^{2}}=\frac{s-s_{2}}{\pi} \int_{R C} d s^{\prime} \frac{\operatorname{Im} t_{4}\left(s^{\prime}\right) / t_{2}\left(s^{\prime}\right)^{2}}{\left(s^{\prime}-s_{2}\right)\left(s^{\prime}-s\right)}+L C\left(t_{4} / t_{2}^{2}\right)+P C\left(t_{4} / t_{2}^{2}\right),
$$

where we have now subtracted at $s_{2}$, which is the LO approximation to the Adler zero, and $P C$ stands for the contribution of the pole at $s_{2}$. We can now use unitarity, Eqs. (1) and (2), to find that the imaginary parts on the right cut of both dispersion relations are exactly opposite to each other. Since the LC integral is weighted at low energies, we can use ChPT to approximate $L C(1 / t) \simeq-L C\left(t_{4} / t_{2}^{2}\right)$. The pole contribution $P C(1 / t)$ can also be evaluated with ChPT since it involves derivatives of $t$ evaluated at the Adler zero, where ChPT is perfectly justified. Finally, we approximate with ChPT $\left(s-s_{A}\right)\left(s^{\prime}-s_{A}\right) \simeq\left(s-s_{2}\right) /\left(s^{\prime}-s_{2}\right)$. Altogether, we find a modified IAM (mIAM) formula:

$$
t^{m I A M}=\frac{t_{2}^{2}}{t_{2}-t_{4}+A^{m I A M}}, \quad A^{m I A M}=t_{4}\left(s_{2}\right)-\frac{\left(s_{2}-s_{A}\right)\left(s-s_{2}\right)\left[t_{2}^{\prime}\left(s_{2}\right)-t_{4}^{\prime}\left(s_{2}\right)\right]}{s-s_{A}} .
$$



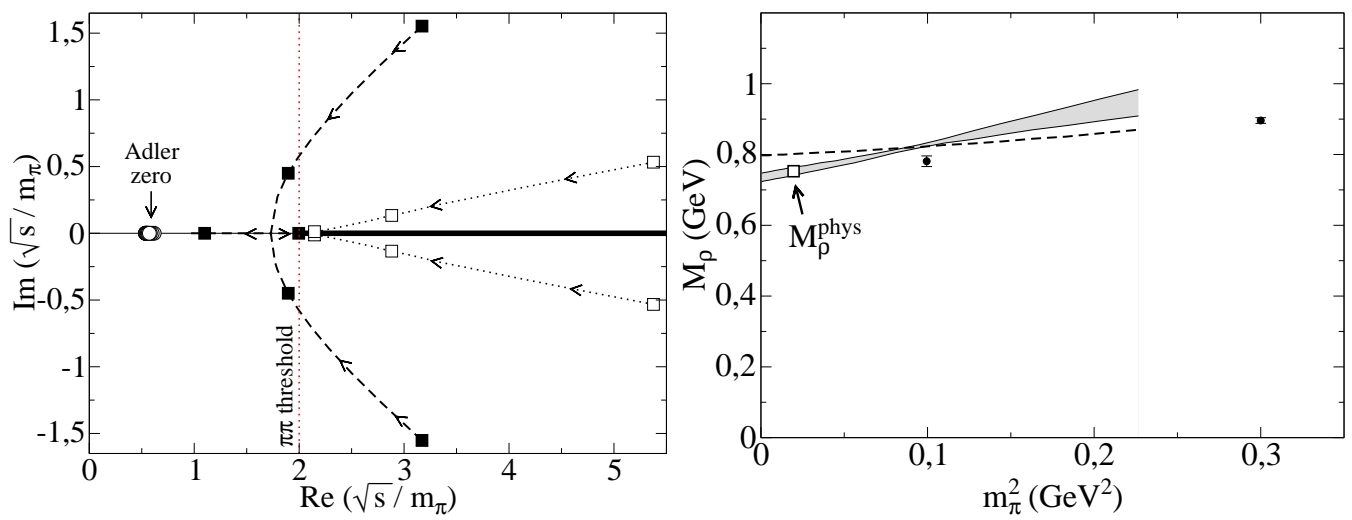

FIGURE 1. Left: Movement of the $\sigma$ (dashed lines) and $\rho$ (dotted lines) poles for increasing $m_{\pi}$ (direction indicated by the arrows) on the second sheet. The filled (open) boxes denote the pole positions for the $\sigma(\rho)$ at pion masses $m_{\pi}=1,2$, and $3 \times m_{\pi}^{\text {phys }}$, respectively. For $m_{\pi}=3 m_{\pi}^{\text {phys }}$ three poles accumulate in the plot very near the $\pi \pi$ threshold. Note that all poles are always far from the Adler zero (circles). Right: The grey band shows the $m_{\pi}$ dependence of $\rho$ pole mass from the IAM versus recent lattice results from [1]. The dashed line is the IAM result for $N_{c}=10$.

The standard IAM is recovered for $A^{m I A M}=0$, which holds exactly for all partial waves except the scalar ones. In the original IAM derivation [7, 8] $A^{m I A M}$ was neglected, since it formally yields a NNLO contribution and is numerically very small, except near the Adler zero, where it diverges. However, if $A^{m I A M}$ is neglected, the IAM Adler zero occurs at $s_{2}$, correct only to LO, it is a double zero instead of a simple one, and a spurious pole of the amplitude appears close to the Adler zero. All of these caveats are removed with the mIAM, Eq. (5). The differences in the physical and resonance region between the IAM and the mIAM are less than $1 \%$. However, as we will see, for large $m_{\pi}$ the $\sigma$ pole splits in two virtual poles below threshold, one of them moving towards zero and approaching the Adler zero region, where the IAM fails. Thus, we will use for our calculations the mIAM, although it is only relevant for the mentioned second $\sigma$ pole, and only when it is very close to the Adler zero.

\section{RESULTS}

By changing $m_{\pi}$ in the amplitudes we see how the poles generated with the IAM evolve. We will use the LECs values $10^{3} l_{3}^{r}=0.8 \pm 3.8$ and $10^{3} l_{4}^{r}=6.2 \pm 5.7$ from [3] and fit the mIAM to data up to the resonance region to find $10^{3} l_{1}^{r}=-3.7 \pm 0.2$ and $10^{3} l_{2}^{r}=5.0 \pm 0.4$. These LECs are evaluated at $\mu=770 \mathrm{MeV}$.

The values of $m_{\pi}$ considered should fall within the ChPT range of applicability and allow for some elastic $\pi \pi$ regime below $K \bar{K}$ threshold. Both criteria are satisfied if $m_{\pi} \leq 500 \mathrm{MeV}$, since $S U$ (3) ChPT still works with such kaon masses, and because for $m_{\pi} \simeq 500 \mathrm{MeV}$, the kaon mass becomes $\simeq 600$, leaving $200 \mathrm{MeV}$ of elastic region.

Fig. 11 (left) shows the evolution of the $\sigma$ and $\rho$ pole positions as $m_{\pi}$ is increased. In order to see the pole movements relative to the two pion threshold, which is also increasing, all quantities are given in units of $m_{\pi}$, so the threshold is fixed at $\sqrt{s}=2$. 
Both poles moves closer to threshold and they approach the real axis. The $\rho$ poles reach the real axis as the same time that they cross threshold. One of them jumps into the first sheet and stays below threshold in the real axis as a bound state, while its conjugate partner remains on the second sheet practically at the very same position as the one in the first. In contrast, the $\sigma$ poles go below threshold with a finite imaginary part before they meet in the real axis, still on the second sheet, becoming virtual states. As $m_{\pi}$ is increased further, one of the poles moves toward threshold and jumps through the branch point to the first sheet and stays in the real axis below threshold, very close to it as $m_{\pi}$ keeps growing. The other $\sigma$ pole moves down in energies further from threshold and remains on the second sheet. This analytic structure, with two very asymmetric poles in different sheets for a scalar wave, could be a signal of a prominent molecular component [10, 11]. Similar pole movements have been also found within quark models [12].

Note that ChPT has ben used to evaluate the subtraction constants at the Adler zero and the low energy part of LC, always far from the resonance poles, even when they move below threshold, as shwon in Fig. 1 (left), being the use of ChPT perfectly justified.

In Fig. 2 (left) we show the $m_{\pi}$ dependence of $M_{\rho}$ and $M_{\sigma}$ (defined from the pole position $\left.\sqrt{s_{\text {pole }}}=M-i \Gamma / 2\right)$, normalized to their physical values. The bands cover the LECs uncertainties. We see that both masses grow with $m_{\pi}$, but $M_{\sigma}$ grows faster than $M_{\rho}$. Below $m_{\pi} \simeq 330 \mathrm{MeV}$ we only show one line because the two conjugate $\sigma$ poles have the same mass. Above $330 \mathrm{MeV}$, these two poles lie on the real axis with two different masses. The heavier pole goes towards threshold and around $m_{\pi} \simeq 465$ moves into the first sheet. Note also that the $m_{\pi}$ dependence of $M_{\sigma}$ is much softer than suggested in [13], shown as the dotted line, which in addition does not show the two virtual poles.

In the right panel of Fig. 2 we show the $m_{\pi}$ dependence of $\Gamma_{\rho}$ and $\Gamma_{\sigma}$ normalized to their physical values, where we see that both widths become smaller. We compare this decrease with the expected reduction from phase space as the resonances approach the $\pi \pi$ threshold. We find that $\Gamma_{\rho}$ follows very well this expected behavior, which implies that the $\rho \pi \pi$ coupling is almost $m_{\pi}$ independent. In contrast $\Gamma_{\sigma}$ shows a different behavior from the phase space reduction expectation. This suggest a strong $m_{\pi}$ dependence of the $\sigma$ coupling to two pions, necessarily present for molecular states [11, 14].

Fig. 1 (right) is a comparison of our results for $M_{\rho}$ with some lattice results [1], which deserves several words of caution. Our $M_{\rho}$ is the "pole mass", which is deep in the complex plane, and, due to the momentum discretization induced by the finite lattice volume, the minimum energy with which pions are produced is larger than the resulting $M_{\rho}$, so the lattice rho has no width. We can mimic in our formalism a narrower $\rho$ by increasing the number of colors, $N_{c}[15]$. We also show the result for the rho mass for $N_{c}=10$. We see that making the $\rho$ artificially narrower yields a better agreement with lattice data. With these caveats in mind our results are in qualitative agreement with those of the lattice. Following [16] one may write $M_{\rho}=M_{\rho}^{0}+c_{1}+O\left(m_{\pi}^{3}\right)$, where the $c_{i}$ parameters are expected to be of order one and $M_{\rho} \sim 0.65-0.80 \mathrm{GeV}$, which is confirmed by a fit to lattice data [16]. We can fit our results and predict $M_{\rho}^{0}=$ $0.735 \pm 0.0017 \mathrm{MeV}$ and $c_{1}=0.90 \pm 0.17 \mathrm{GeV}^{-1}$. Although the pion mass dependence of our calculation is steeper than that of the lattice, the values obtained are still consistent with the expectations mentioned above. Let us remind that the $m_{\pi}$ dependence in our approach is correct only up to NLO in ChPT. 

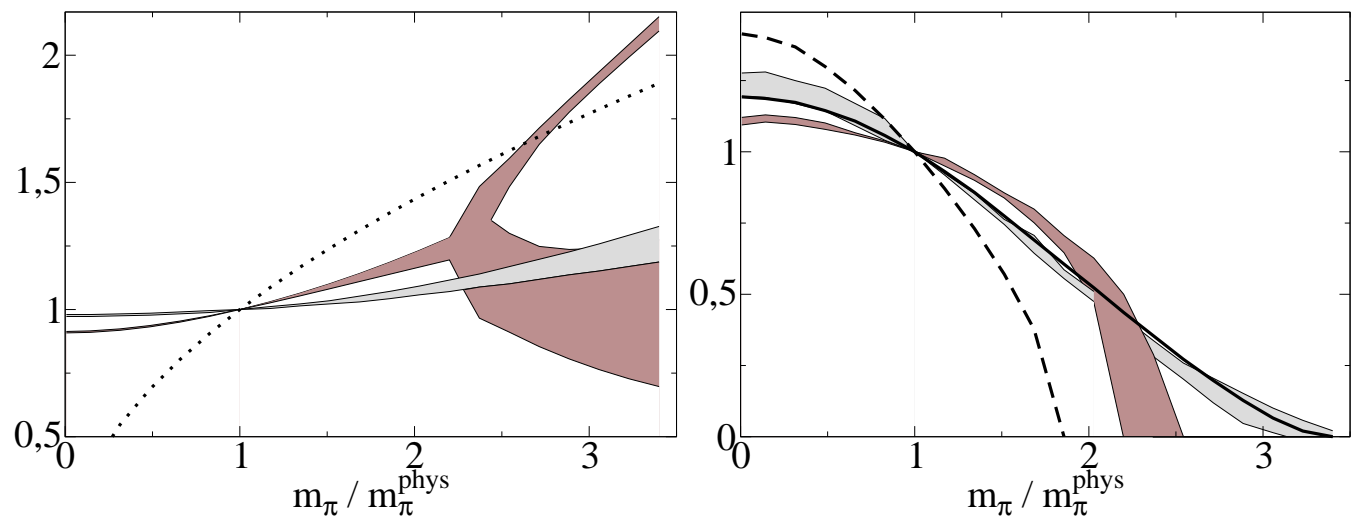

FIGURE 2. $m_{\pi}$ dependence of resonance masses (left) and widths (right) in units of the physical values. In both panels the dark (light) band shows the results for the $\sigma(\rho)$. The width of the bands reflects the uncertainties induced from the uncertainties in the LECs. The dotted line shows the $\sigma$ mass dependence estimated in Ref. [13]. The dashed (continuous) line shows the $m_{\pi}$ dependence of the $\sigma(\rho)$ width from the change of phase space only, assuming a constant coupling of the resonance to $\pi \pi$.

We thank the organicers for creating the nice scientific atmosphere of the workshop and the Spanish research contracts No. PR27/05-13955-BSCH, No. FPA2007-29115E, No. FPA2004-02602, No. UCM-CAM 910309 and No. BFM2003-00856 for partial finantial support.

\section{REFERENCES}

1. S. Aoki et al.,Phys. Rev. D 60, 114508 (1999).

2. K. F. Liu, Prog. Theor. Phys. Suppl. 168, 160 (2007). M. G. Alford and R. L. Jaffe, Nucl. Phys. B 578, 367 (2000) T. Kunihiro et al., Phys. Rev. D 70, 034504 (2004)

3. S. Weinberg, Physica A96 (1979) 327; J. Gasser and H. Leutwyler, Annals Phys. 158 (1984) 142; Nucl. Phys. B 250 (1985) 465.

4. A. Gómez Nicola, J.R. Peláez and G. Ríos, Phys. Rev. D 77 (2008) 056006.

5. C. Hanhart, J. R. Pelaez and G. Rios, arXiv:0801.2871 [hep-ph]. To appear in Phys. Rev. Lett.

6. I. Caprini et al., Phys. Rev. Lett. 96 (2006) 132001

7. T. N. Truong, Phys. Rev. Lett. 61 (1988) 2526. Phys. Rev. Lett. 67, (1991) 2260; A. Dobado et al., Phys. Lett. B235 (1990) 134.

8. A. Dobado and J. R. Peláez, Phys. Rev. D 47 (1993) 4883; Phys. Rev. D 56 (1997) 3057.

9. F. Guerrero and J. A. Oller, Nucl. Phys. B 537 (1999) 459 [Erratum-ibid. B 602 (2001) 641]. J. R. Peláez, Mod. Phys. Lett. A 19, 2879 (2004) A. Gómez Nicola and J. R. Peláez, Phys. Rev. D 65 (2002) 054009 and AIP Conf. Proc. 660 (2003) 102.

10. D. Morgan, Nucl. Phys. A 543 (1992) 632; D. Morgan and M. R. Pennington, Phys. Rev. D 48 (1993) 1185.

11. V. Baru et al., Phys. Lett. B 586 (2004) 53.

12. E. van Beveren et al., AIP Conf. Proc. 660, 353 (2003); Phys. Rev. D 74, 037501 (2006).

13. T. E. Jeltema and M. Sher, Phys. Rev. D 61 (2000) 017301

14. S. Weinberg, Phys. Rev. 130, 776 (1963); Y. Kalashnikova et al., Eur. Phys. J. A 24 (2005) 437.

15. J. R. Peláez and G. Rios, Phys. Rev. Lett. 97 (2006) 242002. J. R. Peláez, Phys. Rev. Lett. 92 (2004) 102001

16. P. C. Bruns and U.-G. Meißner, Eur. Phys. J. C 40, 97 (2005). 